\title{
Studies of jet shapes and substructure with ATLAS
}

\author{
Orel Gueta* ${ }^{*}$ \\ On behalf of the ATLAS collaboration \\ Tel Aviv University \\ E-mail: orel.gueta@cern.ch
}

\begin{abstract}
The internal structure of jets produced in proton-proton collisions at $7 \mathrm{TeV}$ centre-of-mass energy at the LHC provides a direct test of QCD calculations of gluon and quark radiation, and is sensitive to soft QCD effects, such as hadronisation or underlying event. It moreover yields a handle to identify jets coming from boosted hadronic decays of massive particles. The ATLAS collaboration has performed several measurements to characterize jet shapes and substructure observables. The transverse energy distribution around the jet core, as well as the fragmentation of a jet into charged particles is presented. Techniques are studied to reduce the sensitivity of jet properties to soft QCD and to multiple proton-proton collisions. A selection of jet substructure measurement is presented and compared to a range of QCD calculations and phenomenological models.
\end{abstract}

XXI International Workshop on Deep-Inelastic Scattering and Related Subjects 22-26 April, 2013

Marseilles, France

\footnotetext{
* Speaker.

${ }^{\dagger}$ Partly supported by the Israel Science Foundation and German Israel Foundation.
} 


\section{Introduction}

The high center-of-mass energy at the Large Hadron Collider, combined with the coverage and granularity of the ATLAS detector [1], provide an excellent environment to study jets. Jet production is the dominant process in $p p$ collisions and measurements of jet production contribute to the understanding of QCD at high energies. Studies of the internal structure of jets provide a window to investigate both soft and hard QCD phenomena. The internal structure of jets is influenced by fragmentation, hadronisation, colour connections and the underlying event [2]. The substructure of jets may also be used to identify hadronic decays of boosted massive particle [3]. At the LHC, particles such as $W$ bosons $[4,5]$ or top quarks $[6,7]$ are abundantly produced with significant Lorentz boost. At sufficiently large boost, the decay products are collimated such that they are identified as a single jet in the detector. Jets produced by boosted heavy particles are expected to have an invariant mass close to that of the parent particle, where the jet mass is derived from the sum of the four-momenta of all the components of the jet. In addition, such jets have a distinct internal structure which may differentiate between jets produced by heavy particles and those produced by light quarks and gluons.

\section{Jet shapes and fragmentation}

The study of jet shapes in $p p$ collisions provides information about the details of the partonto-jet fragmentation process [8]. ATLAS studied the internal structure of jets [9] in terms of the differential jet shape, $\rho(r)$, and the integrated jet shape, $\Psi(r)$,

$$
\rho(r)=\frac{1}{\Delta r} \frac{1}{N^{\text {jet }}} \sum_{\text {jets }} \frac{p_{T}(r-\Delta r / 2, r+\Delta r / 2)}{p_{T}(0, R)}, \quad \Psi(r)=\frac{1}{N^{\text {jet }}} \sum_{\text {jets }} \frac{p_{T}(0, r)}{p_{T}(0, R)},
$$

where $R$ is the radius of the jet and $r$ is the radial distance from the jet axis, both defined in the rapidity and azimuthal angle plane. Jets are reconstructed from massless calorimeter clusters and the variable $p_{T}(a, b)$ denotes the summed transverse momentum, $p_{T}$, of the clusters between radius $a$ and $b$. Measurements of $\rho(r)$ and $\Psi(r)$ were performed for anti- $k_{t}$ jets [10] with radius parameters of 0.4 and 0.6. The distributions, corrected for detector effects, are compared to a number of predictions from Monte Carlo (MC) generators, as shown in Figure 1. It can be seen that recent tunes of PYTHIA [11 - 14] reproduce well the distributions observed in data. Comparisons with other generators [15] indicate that the predicted jet shapes are mainly dictated by the details on the implementation of the parton showers and the modeling of the underlying event in the MC generators. Similar conclusions were drawn from the comparison of the density of charged particles in a jet and the momentum of charged particles parallel and transverse to the jet axis, between data and MC generators [16].

\section{Jet grooming and substructure}

The jet mass distribution was measured before and after applying a jet grooming procedure on the jet known as splitting and filtering [17]. As an example, for jets clustered with the CambridgeAachen $[18,19]$ algorithm with $R=1.2$, the jet mass distribution before and after applying splitting 


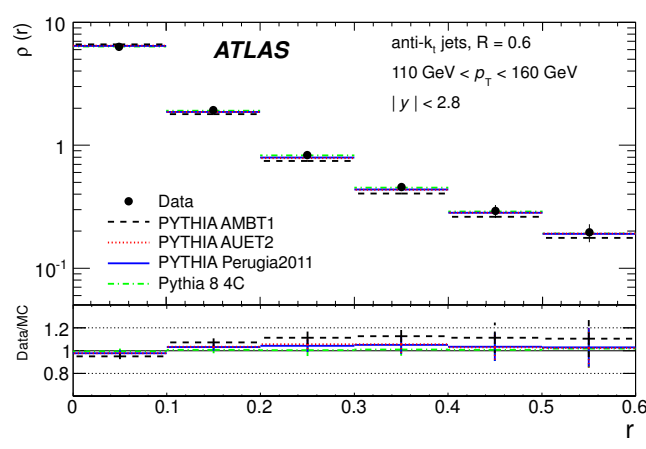

(a)

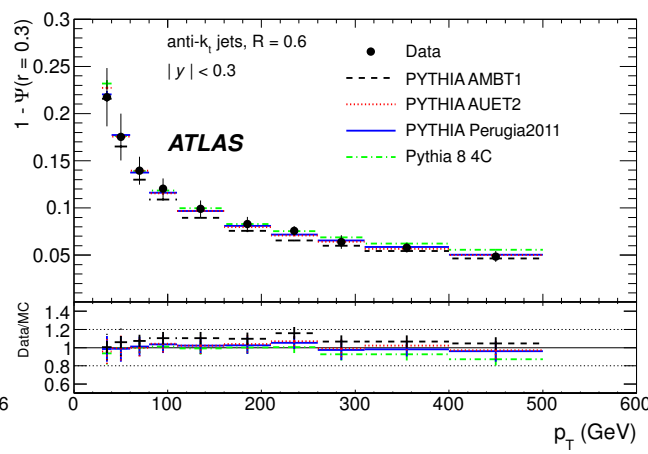

(b)

Figure 1: For anti- $k_{t}$ jets with a radius of 0.6 , (a) the differential jet shape, $\rho(r)$, as a function of the distance from the jet axis, $r$, for jets in the rapidity range, $|y|<2.8$, and transverse momentum, $110<p_{T}<160 \mathrm{GeV}$; (b) the jet shape, $1-\Psi(r)$, where $\Psi(r)$ is the integrated jet shape, as a function of $p_{T}$, for jets in the rapidity range, $|y|<0.3$, compared to PYTHIA tunes, as described in the legend [15]. The lower panels show the ratio between the data and MC expectations. Error bars indicate the statistical and systematic uncertainties added in quadrature.

and filtering is shown in Figure 2 and compared to two MC generators [20]. The MC generators fail to describe the measured jet mass distribution before applying the splitting and filtering procedure, while a much better agreement is achieved after applying the procedure. This implies that the splitting and filtering procedure reduces the sensitivity of the jet mass to soft physics.

Further jet grooming techniques were explored with ATLAS, such as trimming [21]. The effects of trimming were demonstrated on semi-leptonic $t \bar{t}$-candidate events, where one anti- $k_{t}$ jet with $R=0.4$ was tagged as a $b$-jet to reduce background [22]. Figure 3 shows the leading- $p_{T}$ jet mass for anti- $k_{t}$ jets with $R=1.0$ before and after trimming. Trimming moves the mass of quark and gluon jets (likely from a fully-leptonic $t \bar{t}$ event) towards lower masses, while the mass distribution of jets originating from top quarks peaks around the mass of the top.

The dependence of the mean jet mass on the number of simultaneous $p p$ collisions (pileup) is significantly reduced by jet grooming techniques [20,23]. The sensitivity to soft physics may also be reduced by constructing variables such as planar flow, angularity [24], $k_{t}$ splitting scales [20] and $N$-subjettiness [25, 26]. These variables aim to explore the energy distribution inside and on the face of the jet and have the potential to identify jets containing heavy particles. Jet substructure variables and jet grooming techniques were utilized to reduce background in searches for resonances of beyond Standard Model particles [27-30].

\section{Validation of jet mass scale}

Hadronic decays of known heavy particles, where all the decay products are reconstructed as a single jet, may be used to constrain the uncertainty on the response of the calorimeter to jet mass in the ATLAS detector simulation, referred to as the jet mass scale (JMS) uncertainty. The jet mass distribution was measured in events containing a $W \rightarrow \mu v$ candidate and a $b$-tagged jet and the 


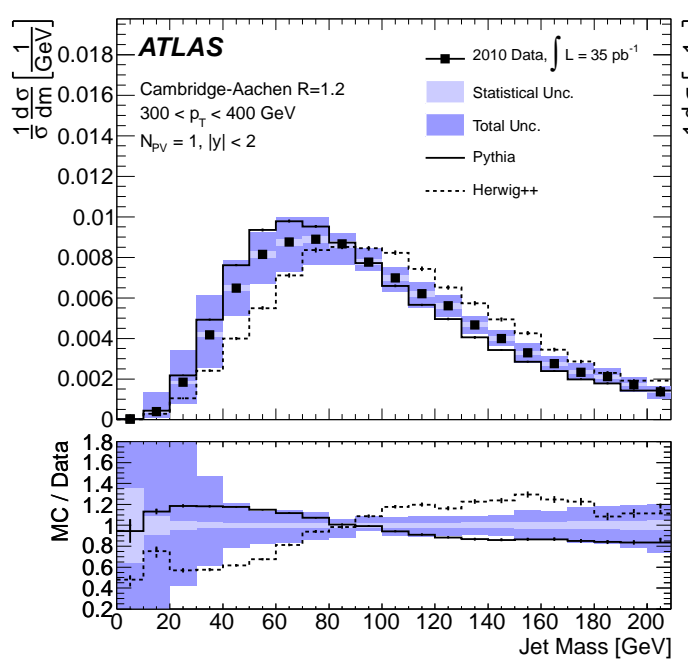

(a)

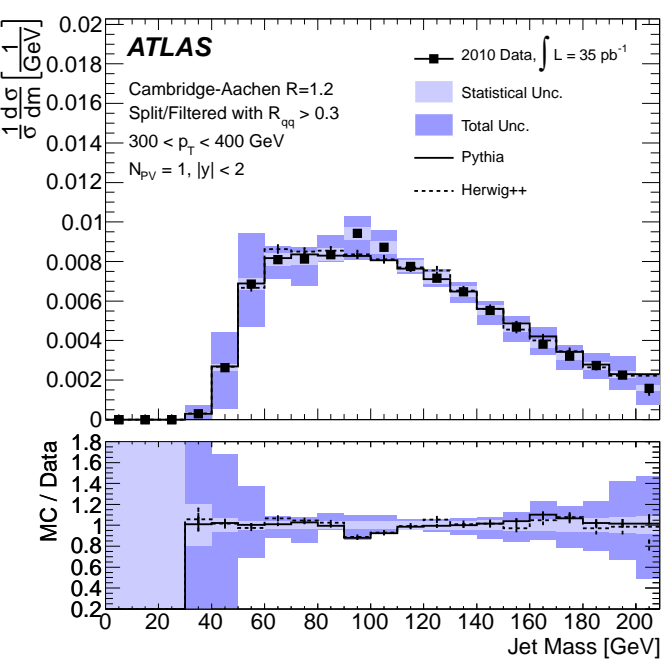

(b)

Figure 2: In events with one primary vertex, a comparison between data, Herwig++ and PYTHIA of normalised cross-sections as a function of jet mass for Cambridge-Aachen jets with $R=1.2$, rapidity in the range $|y|<2$, transverse momentum $300<p_{T}<400 \mathrm{GeV}$, before (a) and after (b) applying splitting and filtering [20]. The lower panels show the ratio between MC expectations and the data. The shaded areas indicate the statistical and systematic uncertainties as described in the legend.

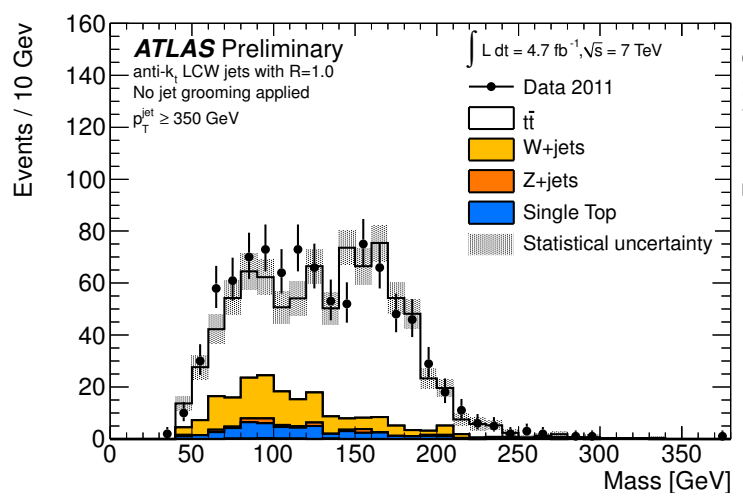

(a)

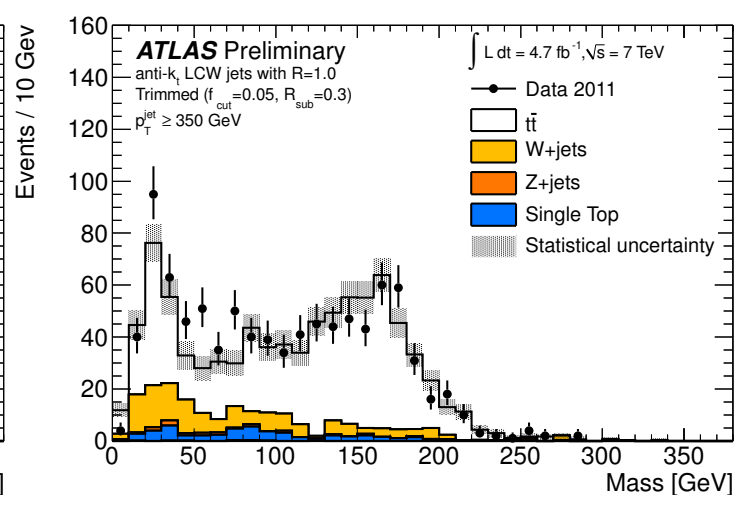

(b)

Figure 3: Leading- $p_{T}$ jet-mass distribution for anti- $k_{t}$ jets with $R=1.0$ and $p_{T} \geq 350 \mathrm{GeV}$ for (a) ungroomed jets and (b) trimmed jets, where one anti- $k_{t}$ jet with $R=0.4$ was tagged as a $b$ jet to reduce background [22]. The distributions from data are compared with MC predictions as described in the legend. Physics backgrounds, also shown, have been added in due proportion to the MC distribution. Error bars indicate the statistical uncertainty. 
location of the mass peak was compared in the data and in the MC for two jet algorithms [31], as shown in Figure 4. In order to reduce background, the splitting and filtering procedure was used on Cambridge-Aachen jets with $R=1.2$ and the trimming procedure was used on anti- $k_{t}$ jets with $R=1.0$. A peak near the $W$ mass is clearly observed for both algorithms in data and simulated MC events. These distributions constrain the JMS uncertainty and provide a data-driven jet-mass calibration.

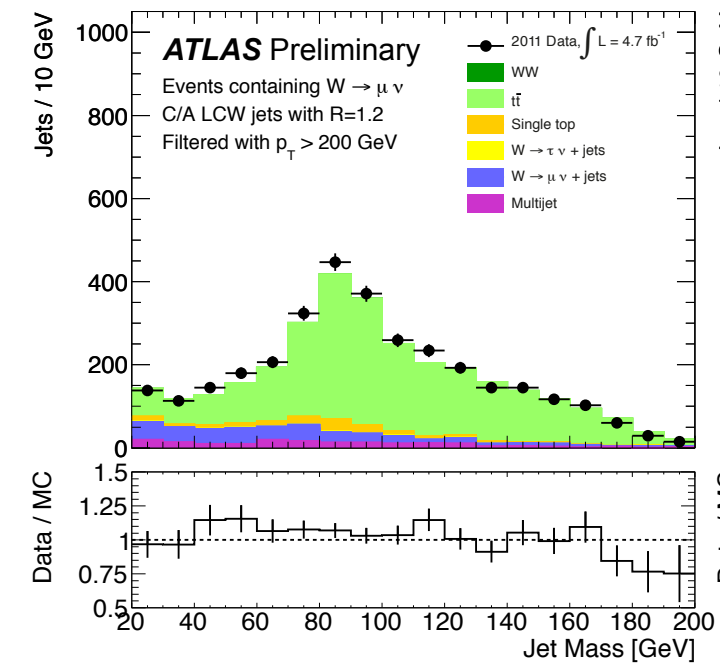

(a)

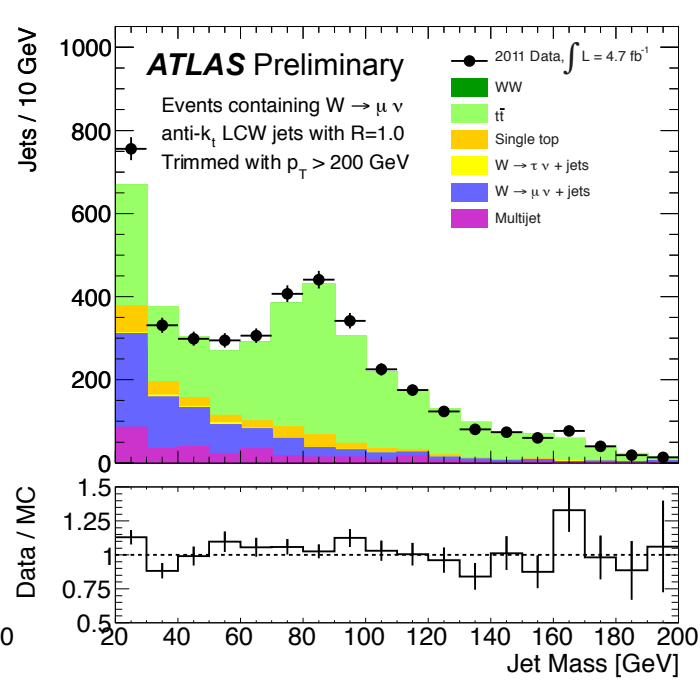

(b)

Figure 4: Jet mass distributions in events containing a $W \rightarrow \mu v$ candidate and a $b$-tagged jet for (a) jets clustered with Cambridge-Aachen with $R=1.2$ and transverse momentum, $p_{T}>200 \mathrm{GeV}$, after splitting and filtering; (b) jets clustered with anti- $k_{t}$ with $R=1.0$ and $p_{T}>200 \mathrm{GeV}$ after trimming [31]. The distributions from data are compared with MC predictions as described in the legend. Physics backgrounds, also shown, have been added in due proportion to the MC distribution. Error bars indicate the statistical uncertainty.

\section{Conclusions}

Jet substructure is used to enhance ATLAS potential for the study of boosted massive particles. ATLAS has measured several observables, including jet shapes, fragmentation functions and transverse profile, jet invariant mass and a number of substructure variables designed for boosted object tagging. Monte Carlo is found to provide an adequate description of most distributions. Jet grooming techniques reduce the soft contamination that affects jet substructure, thus improving the pile-up resilience of jet reconstruction and robustness of the Monte Carlo description. Groomed jet mass distributions have been measured for inclusive jet production, boosted $W$ boson and top quark production. The $W$ boson mass peak is used to constrain the jet mass response of the experiment. 


\section{References}

[1] ATLAS Collaboration, The ATLAS Experiment at the CERN Large Hadron Collider, JINST 3 (2008) S08003.

[2] M. Dasgupta, L. Magnea, and G. P. Salam, Non-perturbative QCD effects in jets at hadron colliders, JHEP 0802 (2008) 055.

[3] A. Abdesselam et al., Boosted objects: A Probe of beyond the Standard Model physics, Eur.Phys.J. C71 (2011) 1661.

[4] M. H. Seymour, Searches for new particles using cone and cluster jet algorithms: A Comparative study, Z. Phys. C62 (1994) 127.

[5] J. Butterworth, B. Cox, and J. R. Forshaw, WW scattering at the CERN LHC, Phys. Rev. D65 (2002) 096014.

[6] ATLAS Collaboration, High $p_{T}$ hadronic top quark identification. Part I: Jet mass and YSplitter, ATL-PHYS-CONF-2008-008 (2008), http://cds.cern.ch/record/1077731.

[7] L. G. Almeida et al., Top Jets at the LHC, Phys. Rev. D79 (2009) 074012.

[8] S. D. Ellis, Z. Kunszt, and D. E. Soper, Jets at hadron colliders at order $\alpha_{s}^{3}$ : A look inside, Phys. Rev. Lett. 69 (1992) 3615.

[9] ATLAS Collaboration, Study of Jet Shapes in Inclusive Jet Production in pp Collisions at $\sqrt{s}=7 \mathrm{TeV}$ using the ATLAS Detector, Phys. Rev. D83 (2011) 052003.

[10] M. Cacciari, G. P. Salam, and G. Soyez, The Anti-k(t) jet clustering algorithm, JHEP 0804 (2008) 063.

[11] ATLAS Collaboration, Charged particle multiplicities in p p interactions at $\sqrt{s}=0.9$ and 7 TeV in a diffractive limited phase-space measured with the ATLAS detector at the LHC and new PYTHIA6 tune, http://cds.cern.ch/record/1277665.

[12] ATLAS Collaboration, New ATLAS event generator tunes to 2010 data, http://inspirehep.net/record/1196773.

[13] P. Z. Skands, Tuning Monte Carlo Generators: The Perugia Tunes, Phys.Rev. D82 (2010) 074018.

[14] R. Corke and T. Sjostrand, Interleaved Parton Showers and Tuning Prospects, JHEP 1103 (2011) 032.

[15] ATLAS Collaboration, Jet Shapes in ATLAS and MC modeling, ATL-PHYS-PUB-2011-010 (2011), https://cds.cern.ch/record/1368881.

[16] ATLAS Collaboration, Measurement of the jet fragmentation function and transverse profile in proton-proton collisions at a center-of-mass energy of $7 \mathrm{TeV}$ with the ATLAS detector, Eur. Phys. J. C71 (2011) 1795.

[17] J. M. Butterworth et al., Jet substructure as a new Higgs search channel at the LHC, Phys. Rev. Lett. 100 (2008) 242001.

[18] Y. L. Dokshitzer et al., Better jet clustering algorithms, JHEP 9708 (1997) 001.

[19] M. Wobisch and T. Wengler, Hadronization corrections to jet cross-sections in deep inelastic scattering, arXiv:hep-ph/9907280 [hep-ph].

[20] ATLAS Collaboration, Jet mass and substructure of inclusive jets in $\sqrt{s}=7$ TeV pp collisions with the ATLAS experiment, JHEP 1205 (2012) 128.

[21] D. Krohn, J. Thaler, and L.-T. Wang, Jet Trimming, JHEP 1002 (2010) 084. 
[22] ATLAS Collaboration, Performance of large-R jets and jet substructure reconstruction with the ATLAS detector, ATLAS-CONF-2012-065 (2012), http://cds.cern.ch/record/1459530.

[23] ATLAS Collaboration, Studies of the impact and mitigation of pile-up on large-R and groomed jets in ATLAS at $\sqrt{s}=7 \mathrm{TeV}$, ATLAS-CONF-2012-066 (2012), http://cds.cern.ch/record/1459531.

[24] ATLAS Collaboration, ATLAS measurements of the properties of jets for boosted particle searches, Phys. Rev. D86 (2012) 072006.

[25] J. Thaler and K. Van Tilburg, Identifying Boosted Objects with N-subjettiness, JHEP 1103 (2011) 015.

[26] J. Thaler and K. Van Tilburg, Maximizing Boosted Top Identification by Minimizing N-subjettiness, JHEP 1202 (2012) 093.

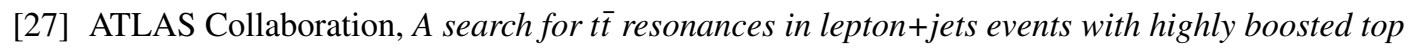
quarks collected in pp collisions at $\sqrt{s}=7 \mathrm{TeV}$ with the ATLAS detector, JHEP 1209 (2012) 041.

[28] ATLAS Collaboration, A search for $t \bar{t}$ resonances in the lepton plus jets final state with ATLAS using $4.7 \mathrm{fb}^{-1}$ of pp collisions at $\sqrt{\mathrm{s}}=7 \mathrm{TeV}$, arXiv:1305.2756 [hep-ex] .

[29] ATLAS Collaboration, A search for $t \bar{t}$ resonances in the lepton plus jets final state with ATLAS using $14 \mathrm{fb}^{-1}$ of pp collisions at $\sqrt{\mathrm{s}}=8 \mathrm{TeV}$, ATLAS-COM-CONF-2013-052 (2013), https://cds.cern.ch/record/1543917.

[30] ATLAS Collaboration, Search for resonances decaying into top-quark pairs using fully hadronic decays in pp collisions with ATLAS at $\sqrt{s}=7$ TeV, JHEP 1301 (2013) 116.

[31] ATLAS Collaboration, Validating the ATLAS Jet Mass Scale with Hadronically Decaying W Bosons, https://twiki.cern.ch/twiki/bin/view/AtlasPublic/JetEtmissApproved2013Substructure. 